\title{
Multiple Sexual Partners as a Potential Independent Risk Factor for Cervical Cancer: a Meta-analysis of Epidemiological Studies
}

\author{
Zhi-Chang Liu ${ }^{1}$, Wei-Dong Liu ${ }^{1}$, Yan-Hui Liu ${ }^{2}$, Xiao-Hua Ye ${ }^{1 *}$, Si-Dong Chen ${ }^{1 *}$
}

\begin{abstract}
It's known that having multiple sexual partners is one of the risk factors of human papillomavirus (HPV) infection which is a major cause of cervical cancer. However, it is not clear whether the number of sexual partners is an independent risk factor for cervical cancer. We identified relevant studies by searching the databases of MEDLINE, PubMed and ScienceDirect published in English from January 1980 to January 2014. We analyzed those studies by combining the study-specific odds ratios (ORs) using random-effects models. Forty-one studies were included in this meta-analysis. We observed that the number of sexual partners was associated with the occurrence of non-malignant cervical disease $(\mathrm{OR}=1.82$, 95\% CI 1.63-2.00) and invasive cervical carcinoma $(\mathrm{OR}=1.77,95 \% \mathrm{CI}$ 1.50-2.05). Subgroup analyses revealed that the association remained significant after controlling for HPV infection (OR=1.52, 95\% CI 1.21-1.83 for non-malignant disease; $O R=1.53,95 \%$ CI 1.301.76 for invasive cervical carcinoma). We found that there was a non-linear relation of the number of sexual partners with both non-malignant cervical disease and invasive cervical carcinoma. The risk of both malignant and non-malignant disease is relatively stable in women with more than 4-7 sexual partners. Furthermore, the frequency-risk of disease remained significant after controlling for HPV infection.The study suggested that having multiple sexual partners, with or without HPV infection, is a potential risk factor of cervical cancer.
\end{abstract}

Keywords: Multiple sexual partners - cervical cancer - meta-analysis - HPV infection

Asian Pac J Cancer Prev, 16 (9), 3893-3900

\section{Introduction}

Cervical cancer is the second most common cancer and an important public health problem worldwide. It is generally known that HPV infection is one of the major causes of cervical cancer (Kjaer et al., 1996a; Walboomers et al., 1999). After the discovery of the strong link between HPV infection and cervical cancer, it remains unclear and controversial whether the associations of other known risk factors for cervical cancer are still relative or have become irrelevant. In addition, with the development of HPV vaccines, most vaccinating women will be protected from the infection. However, HPV is not found in all cases of this neoplasm, and most HPV-positive patients have not proceeded to cervical cancer. Therefore, it is necessary to investigate what are the other important risk factors of cervical cancer.

Epidemiological studies consistently show that sexual behavior is the dominant risk factor of HPV infection, especially in those with multiple sexual partners. A pooled analysis of the international Agency for Research on Cancer (Vaccarella et al., 2006) had confirmed that the number of sexual partners was an important risk factor for HPV infection. However, whether the number of sexual partners is the risk factor of cervical cancer is not well documented. Some previous studies have shown that the number of sexual partners is an independent factor of cervical cancer after adjusted for HPV infection (Herrero et al., 1990; Wang and Lin, 1996). However, other studies did not show the same independent effect (Millikan, 1994; Kjaer et al., 1996b). A collaborative reanalysis (2009) has discussed the relationship between cervical cancer and sexual behavior, including the number of sexual partners and the age at first intercourse (AFI). Because of the limited information on HPV status, the relationship between the number of sexual partners and cervical cancer remains unclear. Therefore, we decided to explore the relationship between the number of sexual partners and the risk of cervical cancer through this meta-analysis.

\section{Materials and Methods}

\section{Literature search strategy}

The meta-analysis was conducted following the 
PRISMA guidelines (Moher, Liberati, Tetzlaff and Altman, 2010). A systematic literature search was performed in the database of MEDLINE, PubMed and ScienceDirect to identify epidemiological studies published in English from January 1980 to January 2014. The search used any combination of the keywords: (cervical cancer OR cervical carcinoma OR cervical neoplasm OR cervical neoplasia OR carcinoma in situ OR uterine cervix cancer) and ('sexual partner' OR 'multiple partners') and ('odds ratio' OR 'relative risk' OR 'hazard ratio'). In addition, we reviewed the content pages of the major epidemiological journals and the reference lists of relevant review articles.

\section{Inclusion and exclusion criteria}

Studies were included if they meet the following criteria: 1) case-control or cohort design, published as fulltext manuscripts; 2) provide information on the number of sexual partners in relation to cervical cancer, the number of cases $\geq 50 ; 3$ ) the patients were definitely diagnosed as cervical intraepithelial neoplasia (CIN) or squamous intraepithelial lesion (SIL) or atypical glandular cells of undetermined significance (ASCUS) or carcinoma in situ of the cervix (CIS) or invasive cervical carcinoma (ICC). The control population has no related diseases; 4) provide original data and include odds ratios (ORs), relative risks (RRs) or hazard ratios (HRs) and their 95\% confidence intervals (CI). Studies were excluded if: 1) they were not published as full reports, such as conference abstracts and letters to editors; 2) they were based on selected patients with specific population (such as HPV-positive or HIVpositive population); 3) the data was combined or analyzed with two types of neoplasm. If an article has included multiple studies, we selected each individual study. If multiple reports were published on the same population or subpopulation, we selected in the meta-analysis only the most recent and detailed one.

\section{Data extraction}

Data extracted from each study included the name of the first author, publication year, region, age, number of subjects, type of neoplasm, adjustments, outcome measures, number of sexual partners, estimates of cancer and corresponding $95 \% \mathrm{CI}$. We chose the estimates of the number of lifetime partners, instead of the number of regular partners, number of recent partners or the number of extra-marital partners, because it is more representative and common. If separate risk estimates were available for different locations or each grade of CIN and their cases were more than 30 , we included both risk estimates in this meta-analysis because they were based on independent cases. The incidence rate of cervical cancer was estimated being less than 5\% (McDougall et al., 2007), OR was used to refer to all risk estimates including RRs, ORs or HRs approximately. Three investigators (Z-C Liu, W-D Liu and Y-H Liu) independently reviewed and cross-checked the data. Any disagreements were resolved by consensus.

The study quality was assessed by using the NewcastleOttawa Scale (NOS), a validated quality assessment instrument for non-randomized trials. The maximum quality score is nine points and the minimum is zero.
Studies with score of eight or greater were regarded as low risk studies, whereas those with a NOS score of less than four were considered as high risk studies.

\section{Statistical analysis}

Those including studies were subdivided into two groups (non-malignant cervical disease and ICC) depending on the case definition. The non-malignant cervical diseases included CIN/SIL, CIS and ASCUS. For some studies, the estimate for the number of partners was computed by pooling the ORs for various categories. We defined reference category as few partners. Other categories, which were more than the reference category, were regarded as multiple partners and computed their combined ORs of multiple partners and $95 \%$ CI from the estimates reported in each study by using a random-effects model. In some studies, the risk estimates of different histological subtypes of ICC were provided separately. We combined their estimates by using random-effects model. Whenever possible, we computed summary estimates for different number of partners. Secondly, subgroup analysis was performed according to study design [cohort study (including the nested case-control study) or case-control study], sample source (populationbased or hospital-based), geographical region, subtype and adjustments for covariates [including HPV, AFI and reproductive factors (including age at first pregnancy and number of pregnancies)] to explore the risk of cervical cancer in each subgroup. A meta-regression analysis was used to investigate whether the heterogeneities were significant ( $p<0.1$ was considered significant). In a further sub-analysis, we investigated the difference between the number of partners and CIN stratified by grade of CIN. To derive the frequency-risk relation between the number of partners and cervical cancer, we used a random-effect linear trend regression and a restricted cubic spline regression (Stata GLST command) to choose the best fitted model (Orsini, Li, Wolk, Khudyakov and Spiegelman, 2012). Because very few studies had categorized above 10 partners, we combined the estimates for all those having 10 partners in each studies to avoid a false conduct. When intervals of the number of partners' categories were reported, we chose the midpoint between the upper and lower levels in the categories. For the open-ended upper interval, we chose $20 \%$ higher than the lower end of the interval to derive the midpoint (Ye et al., 2013).

We calculated the Q-statistic ( $p<0.1$ was considered significant) and the $\mathrm{I}^{2}$ value to test for statistical heterogeneity between studies. $\mathrm{I}^{2}$ ranges of $25 \%-50 \%$, $50 \%-75 \%$, and $\geq 75 \%$ was considered to represent low, moderate, and high heterogeneity respectively (Higgins and Thompson, 2002; Higgins, Thompson, Deeks and Altman, 2003). Publication bias $(p<0.1$ was considered significant) was examined by using a funnel plot, Egger's test and Begg's test (Begg and Mazumdar, 1994; Egger, Davey, Schneider and Minder, 1997). Furthermore, we conducted a sensitivity analysis by sequential omission of individual studies and excluding the high risk studies. All statistical analyses were performed using the STATA (version 12.0). 


\section{Results}

\section{Characteristics of the studies}

The literature search and study selection process were shown in Figure 1. We initially identified 1165 potentially relevant articles. After scanning of the titles and abstracts, 1088 studies were excluded. Through reading the full text of the remaining studies, another 36 studies were excluded. Finally, only 41 articles were included in the meta-analysis. These articles included 21 studies of non-malignant cervical disease, and 23 studies of ICC. In the 41 studies, three studies (Kjaer, 1998; Thomas et al., 2001; Kanjanavirojkul, Pairojkul, Yuenyao and Patarapadungkit, 2006) have provided information of non-malignant cervical disease and ICC, respectively. Four studies (La Vecchia, Negri, Fedele, Franceschi and Gallotta, 1992; Brisson et al., 1994; Parazzini, Velema et

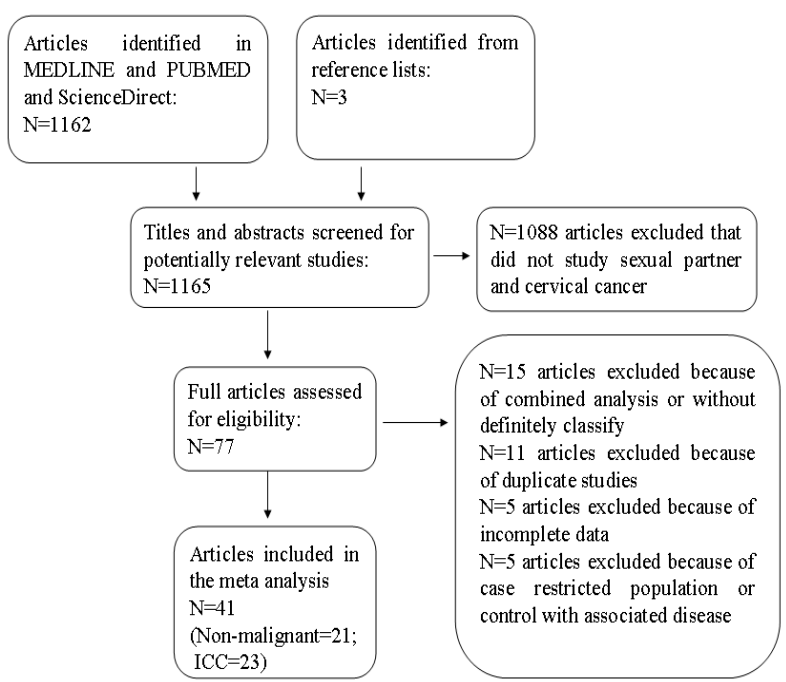

Figure 1. Selection of Studies for Inclusion in the Meta-analysis

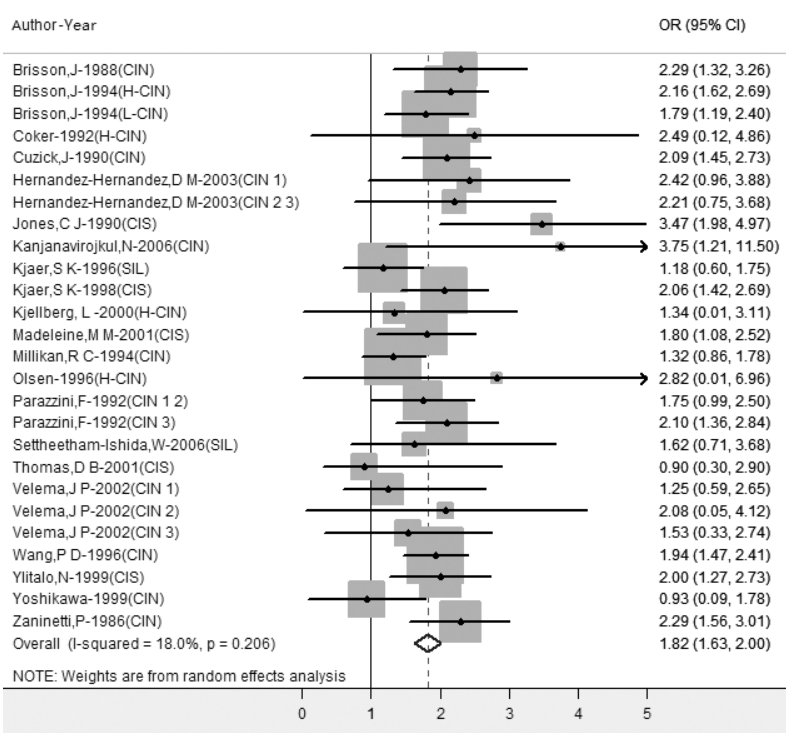

Figure 2. Forest Plot Corresponding to the Random Effects Meta-Analysis Summarizing the Relation Between the Number of Sexual Partners and the Risk of Non-Malignant Cervical Disease; $R R=$ relative risk; CI=confidence interval al., 2002; Hernandez-Hernandez et al., 2003) were about the non-malignant cervical disease reported separate risk estimates for different grades of CIN and one study (Bosch et al., 1992) reported two separate risk estimates for women in Spain and Colombia. We included both of these risk estimates. Thus, this meta-analysis included 26 risk estimates from 5547 cases of non-malignant cervical disease and 24 risk estimates from 6446 cases of ICC. Based on the NOS score, six articles provided information of low risk cases and six articles with high risk cases, while all other studies provided information of middle risk cases.

\section{Non-malignant cervical disease}

The overall estimate for the relationship of nonmalignant disease with the number of sexual partners (multiple partners versus few partners) was 1.82 (95\% CIs 1.63-2.00; Figure 2), and mild heterogeneity was observed ( $\mathrm{P}$ value for heterogeneity $=0.21, \mathrm{I}^{2}=18.0 \%$ ). The number of sexual partners' midpoint were categorized at 2-3,3.1-5, 5.1-9.9 and $\geq 10$ partners, the estimates were 1.61 (95\% CI

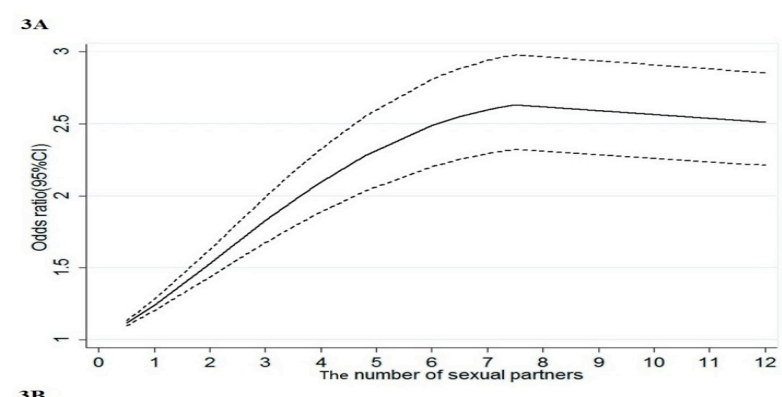

3B

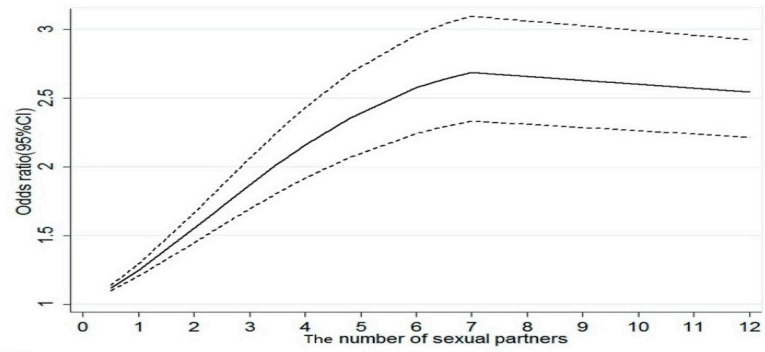
$3 c$

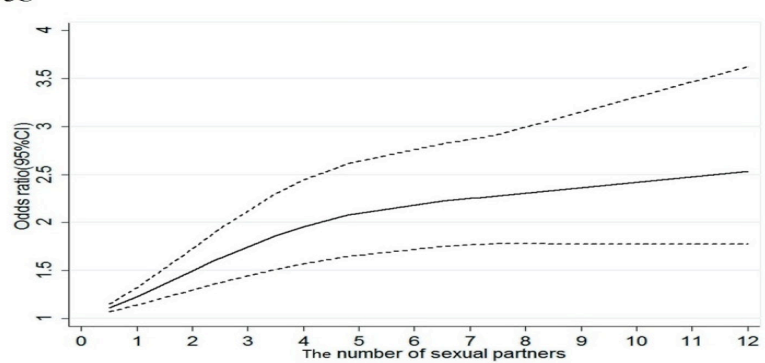

Figure 3. (A) Combined RR (95\% CI) of NonMalignant Cervical Disease and Test for the NonLinearity of the Association Using the Random-Effect Restricted Cubic Spline Model with 3 Knots $(0.75,2.5$, And 12). $P$ for Non-Linearity $<\mathbf{0 . 0 0 1}$. (B) Combined RR $(95 \% \mathrm{CI})$ of non-malignant cervical disease that did not adjusted for HPV infection and test for the linearity of the association using the random-effect restricted cubic spline model with 3 knots $(1,2.5$, and 12). P for non-linearity $<0.001$. (C) Combined $\mathrm{RR}(95 \% \mathrm{CI})$ of non-malignant cervical disease after controlling for HPV infection and test for the linearity of the association using the random-effect restricted cubic spline model with 3 knots $(1,2.5$, and 7.2). P for non-linearity $<0.001$ 


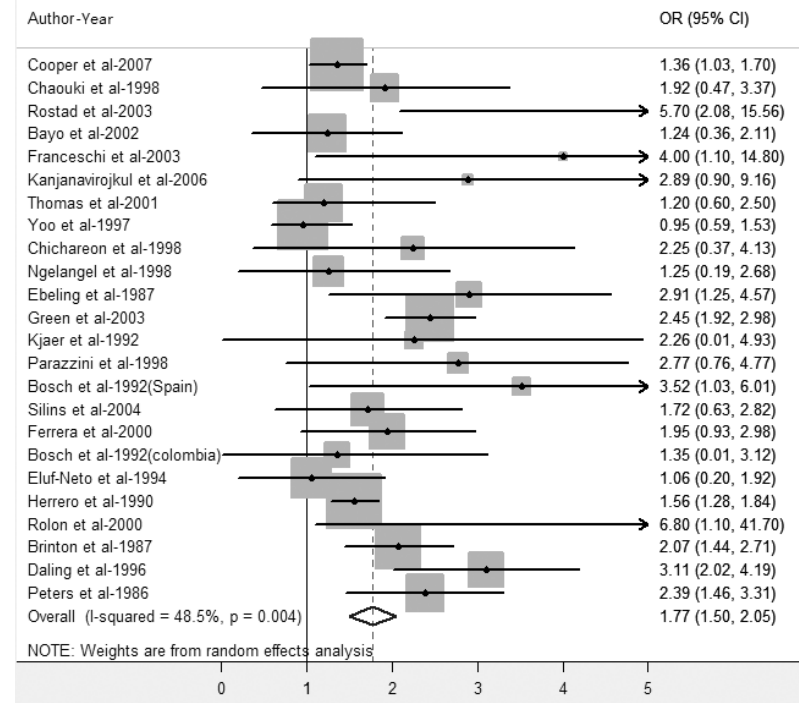

Figure 4. Forest Plot Corresponding to the Random Effects Meta-Analysis Summarizing the Relationship Between the Number of Sexual Partners and the Risk of Invasive Cervical Carcinoma; RR=relative risk; CI=Confidence Interval

$\left.1.36-1.85, \mathrm{I}^{2}=0.0\right), 1.85\left(95 \% \mathrm{CI} 1.46-2.24, \mathrm{I}^{2}=19.7 \%\right)$, $2.12\left(95 \%\right.$ CI $\left.1.71-2.53, \mathrm{I}^{2}=0.0 \%\right)$, and $2.25(95 \%$ CI $1.83-$ $2.67, \mathrm{I}^{2}=0.0 \%$ ), respectively.

A significant heterogeneity was observed in cohort studies compared with case-control studies ( $\mathrm{P}$ value for difference $=0.03$; Table 1), with pooled ORs for cohort studies were 1.44 (95\% CI 1.01-1.86) and pooled ORs for case-control studies were 1.93 (95\% CI 1.73-2.12). We found that the association differed by adjustment for HPV infection $(p=0.06)$. Pooled ORs were $1.52(95 \% \mathrm{CI}$ 1.21-1.83) and 1.99 (95\% CI 1.79-2.19) for those studies adjusted and unadjusted for HPV infection, respectively. When we examined the associations by sample source, geographical region, subtype, adjustment for AFI, and adjustment for reproductive factors, the associations were not significantly different.

There were four studies provided data stratified by grade of CIN (Parazzini et al., 1992; Brisson et al., 1994; Velema et al., 2002; Hernandez-Hernandez et al., 2003). Three studies did not included CIN 1 cases (Coker, Rosenberg, McCann and Hulka, 1992; Olsen, Dillner, Gjoen, Sauer, Orstavik and Magnus, 1996; Kjellberg et al., 2000). No difference was found between high-grade CIN (including CIN 2 and 3;n=8) and low-grade CIN (including CIN 1 and CIN 1,2;n=4) based on the analysis on ORs.

There was no evidence of publication bias as indicated by the analysis using funnel plot, Egger's test ( $\mathrm{P}$ value for bias $=0.43$ ), and Begg's test ( $\mathrm{P}$ value for bias $=0.31$ ). Omission of one study at a time did not impact the overall results. The summary ORs ranged from 1.79 (95\%CI $1.62-1.96)$ to 1.87 (95\% CI 1.69-2.05). And it altered slightly after excluded the studies with high risk (Parazzini et al., 1992; Brisson et al., 1994; Brisson, Roy, Fortier, Bouchard and Meisels, 1988; Kanjanavirojkul et al., 2006) (OR=1.77, 95\%CI 1.51-2.02). We also explore the stability of the studies that had adjusted for the status
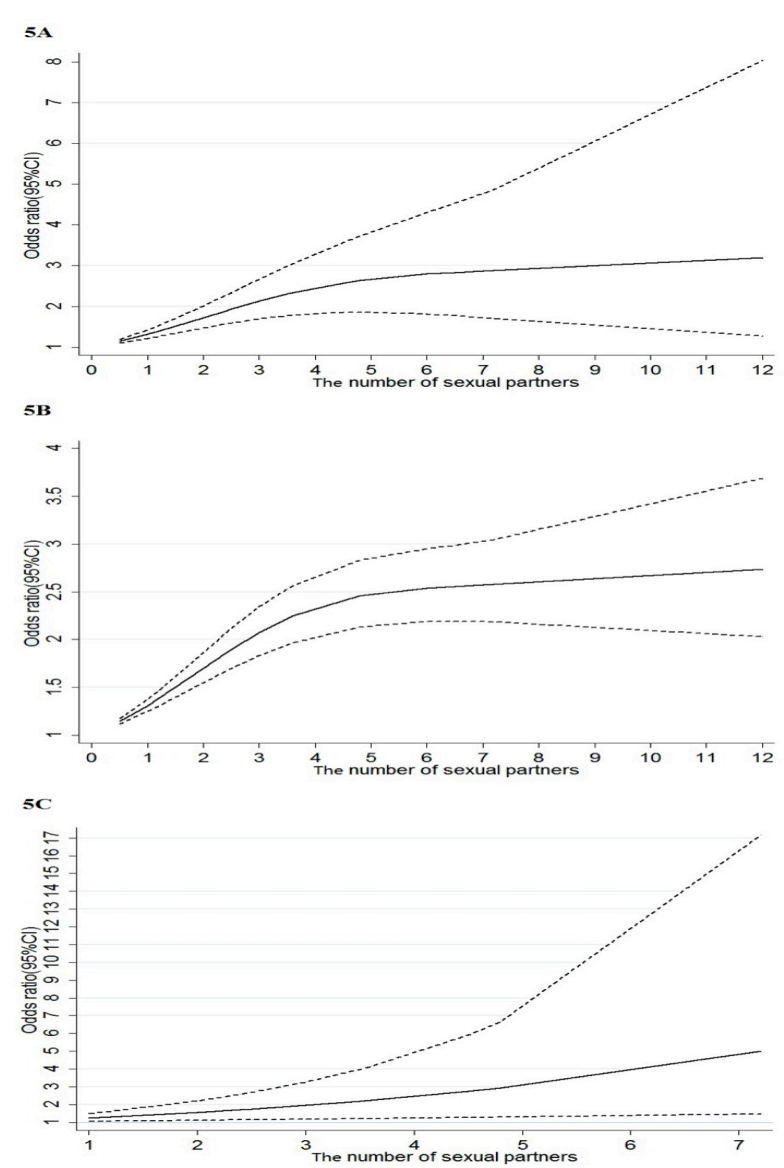

Figure 5. (A) Combined RR (95\% CI) of Invasive Cervical Carcinoma and Test for the Non-Linearity of the Association Using the Random-Effect Restricted Cubic Spline Model with 3 Knots (1, 2.5, And 6.9). $\mathbf{P}$ for Non-Linearity $<\mathbf{0 . 0 0 1}$. (B) Combined RR $(95 \% \mathrm{CI})$ of invasive cervical carcinoma that did not adjusted for HPV infection and test for the linearity of the association using the random-effect restricted cubic spline model with 3 knots (1, 2.5, and 6.5). P for non-linearity $<0.001$. (C) Combined RR $(95 \% \mathrm{CI})$ of invasive cervical carcinoma after controlling for HPV infection and test for the linearity of the association using the random-effect linear trend regression. $P$ for linearity $=0.007$

of HPV infection by using sensitivity analysis. There was no significant change found in the estimates.

We observed a non-linear relation between the number of sexual partners and non-malignant cervical disease ( $\mathrm{P}$ for non-linearity $<0.001$; Figure $3 \mathrm{~A}$ ). The risk of non-malignant cervical disease did not increase when the number of sexual partners was over 7 . We further explored whether there is a change of the trend of the risk after controlling for HPV infection. A similar non-linear relation was found in the studies that did not adjust for HPV infection (P for non-linearity $<0.001$, Figure 3B). However, after controlling for HPV infection, there was a partial change in the trend of risk. The risk of disease increased in women who had more than 7 sexual partners ( $\mathrm{P}$ for non-linearity $=0.007$; Figure 3C).

\section{Invasive cervical carcinoma}

Women with multiple sexual partners showed a significant increase of risk of developing ICC than those who has only few partners $(\mathrm{OR}=1.77,95 \% \mathrm{CIs}$ 1.50-2.05; Figure 4). We detected some heterogeneity 
Multiple Sexual Partners as an Independent Risk Factor for Cervical Cancer: a Meta-Analysis

Table 1. Subgroup Results of the Number of Sexual Partner Associated with Non-Malignant Cervical Disease

\begin{tabular}{|c|c|c|c|c|}
\hline Subgroups & $\begin{array}{l}\text { Stratification criterion } \\
\text { (Number of OR) }\end{array}$ & $\begin{array}{l}\mathrm{RR}(95 \% \mathrm{CI}) \\
\text { multiple vs. few }\end{array}$ & $\mathrm{I}^{2}$ & $\begin{array}{c}\mathrm{P} \text { a value } \\
\text { for difference }\end{array}$ \\
\hline All studies & Non-malignant cervical disease(26) & $1.82(1.63,2.00)$ & $18.0 \%$ & \\
\hline Study design & $\begin{array}{l}\text { Cohort (3) } \\
\text { Case-control (23) }\end{array}$ & $\begin{array}{l}1.44(1.01,1.86) \\
1.93(1.75,2.11)\end{array}$ & $\begin{array}{r}38.9 \% \\
0.0 \%\end{array}$ & $p=0.03$ \\
\hline Sample source & $\begin{array}{l}\text { Population (14) } \\
\text { Hospital (12) }\end{array}$ & $\begin{array}{l}1.70(1.46,1.94) \\
2.02(1.75,2.30)\end{array}$ & $\begin{array}{r}25.4 \% \\
0.0 \%\end{array}$ & $p=0.14$ \\
\hline Geographical region & $\begin{array}{l}\text { Asia (5) } \\
\text { Latin (5) } \\
\text { Europe (9) } \\
\text { USA+ Canada (7) }\end{array}$ & $\begin{array}{l}1.49(0.90,2.08) \\
1.75(1.15,2.34) \\
1.88(1.61,2.15) \\
1.94(1.53,2.36)\end{array}$ & $\begin{array}{r}35.2 \% \\
0.0 \% \\
10.6 \% \\
50.0 \%\end{array}$ & $\begin{array}{l}\text { Reference } \\
\qquad \begin{array}{r}p=0.99 \\
p=0.67 \\
p=0.64\end{array}\end{array}$ \\
\hline Type of cancer & $\begin{array}{l}\text { CIS (5) } \\
\text { CIN/SIL (21) }\end{array}$ & $\begin{array}{l}1.98(1.47,2.49) \\
1.78(1.58,1.97)\end{array}$ & $\begin{array}{l}40.9 \% \\
12.3 \%\end{array}$ & $p=0.38$ \\
\hline & $\begin{array}{l}\text { High-grade CIN (8) } \\
\text { Low-grade CIN (4) }\end{array}$ & $\begin{array}{l}2.06(1.69,2.42) \\
1.74(1.33,2.15)\end{array}$ & $\begin{array}{l}0.0 \% \\
0.0 \%\end{array}$ & $p=0.30 \mathrm{~b}$ \\
\hline Adjustment for HPV & $\begin{array}{l}\text { Yes }(9) \\
\text { No }(17)\end{array}$ & $\begin{array}{l}1.52(1.21,1.83) \\
1.99(1.79,2.19)\end{array}$ & $\begin{array}{r}19.4 \% \\
0.0 \%\end{array}$ & $p=0.06$ \\
\hline Adjustment for AFI & $\begin{array}{l}\text { Yes }(6) \\
\text { No }(20)\end{array}$ & $\begin{array}{l}2.01(1.74,2.28) \\
1.74(1.49,1.98)\end{array}$ & $\begin{array}{r}0.0 \% \\
26.4 \%\end{array}$ & $p=0.64$ \\
\hline Adjustment for reproductive factors & $\begin{array}{l}\text { Yes (3) } \\
\text { No (23) }\end{array}$ & $\begin{array}{l}1.99(1.65,2.32) \\
1.78(1.56,2.00)\end{array}$ & $\begin{array}{r}0.0 \% \\
24.1 \%\end{array}$ & $p=0.89$ \\
\hline
\end{tabular}

*CIN=cervical intraepithelial neoplasia. $\mathrm{CIS}=$ carcinoma in situ. $\mathrm{SIL}=$ squamous intraepithelial lesion. $\mathrm{RR}=\mathrm{Relative}$ risk. $\mathrm{CI}=$ confidence interval . $\mathrm{HPV}=$ human papillomavirus. AFI=age at first intercourse. Reproductive factors included age at first pregnancy and number of pregnancies. a The $p$ values for difference across strata were obtained by using meta-regression. b Comparing high-grade CIN with low-grade CIN

Table 2. Subgroup Results of the Number of Sexual Partner Associated with Invasive Cervical Cancer

\begin{tabular}{|c|c|c|c|c|}
\hline Subgroups & $\begin{array}{l}\text { Stratification criterion } \\
\text { (Number of included) }\end{array}$ & $\begin{array}{l}\mathrm{RR}(95 \% \mathrm{CI}) \\
\text { multiple } v s . \text { few }\end{array}$ & $\mathrm{I}^{2}$ & $\begin{array}{l}\mathrm{P} \text { a value for } \\
\text { difference }\end{array}$ \\
\hline All studies & ICC (24) & $1.77(1.50,2.10)$ & $48.5 \%$ & \multirow{3}{*}{$p=0.40$} \\
\hline \multirow[t]{2}{*}{ Sample source } & Population (9) & $2.11(1.59,2.63)$ & $63.5 \%$ & \\
\hline & Hospital (15) & $1.51(1.22,1.81)$ & $23.7 \%$ & \\
\hline \multirow[t]{5}{*}{ Geographical region } & USA (3) & $2.39(1.83,2.96)$ & $24.2 \%$ & \multirow{2}{*}{$\begin{array}{l}\text { Reference } \\
p=0.92\end{array}$} \\
\hline & Europe (6) & $2.41(1.98,2.84)$ & $0.0 \%$ & \\
\hline & Asia (6) & $1.10(0.72,1.49)$ & $0.0 \%$ & $p=0.02$ \\
\hline & Africa (4) & $1.38(1.07,1.69)$ & $0.0 \%$ & $p=0.04$ \\
\hline & Latin America (5) & $1.54(1.28,1.79)$ & $0.0 \%$ & $p=0.04$ \\
\hline \multirow[t]{2}{*}{ Adjustment for HPV } & Yes (10) & $1.53(1.30,1.76)$ & $0.0 \%$ & \multirow[t]{2}{*}{$p=0.39$} \\
\hline & No (14) & $2.00(1.55,2.45)$ & $65.9 \%$ & \\
\hline \multirow[t]{2}{*}{ Adjustment for AFI } & Yes (12) & $1.70(1.34,2.06)$ & $67.9 \%$ & \multirow[t]{2}{*}{$p=0.23$} \\
\hline & No (12) & $1.94(1.51,2.36)$ & $0.0 \%$ & \\
\hline \multirow[t]{2}{*}{ Adjustment for reproductive factors } & Yes (10) & $1.84(1.36,2.32)$ & $60.4 \%$ & \multirow[t]{2}{*}{$p=0.92$} \\
\hline & No (14) & $1.76(1.40,2.13)$ & $40.0 \%$ & \\
\hline
\end{tabular}

*ICC=invasive cervical carcinoma. $\mathrm{RR}=$ Relative risk. $\mathrm{CI}=$ confidence interval. $\mathrm{HPV}=$ human papillomavirus. $\mathrm{AFI}=$ age at first intercourse. Reproductive factors included age at first pregnancy and number of pregnancies. a The $p$ values for difference across strata were obtained by using meta-regression

between studies $\left(p=0.004, \mathrm{I}^{2}=48.5 \%\right)$. The number of sexual partners' midpoint categorized at 2-3, 3.1-5, and $\geq 5.1$ partners, the risk of ICC increased and the estimates were significant $\left[\left(\mathrm{OR}=1.61,95 \% \mathrm{CI} 1.33-1.88, \mathrm{I}^{2}=29.4 \%\right)\right.$; $\left(\mathrm{OR}=2.01,95 \% \mathrm{CI} 1.48-2.55, \mathrm{I}^{2}=48.0 \%\right) ;(\mathrm{OR}=2.40$, 95\% CI $\left.1.95-2.85, \mathrm{I}^{2}=0.0 \%\right)$, respectively].

In subgroup analyses, we found that there was a significant difference in the variable of regions ( $P$ $<0.05$, Table 2). The summary ORs of 2.39 (95\% CI 1.832.96), 2.41 (95\%CI 1.98-2.84), 1.10 (95\%CI 0.72-1.49), 1.38 (95\% CI 1.07-1.69) and 1.54 (95\% CI 1.28-1.79) for studies were found in studies conducted in USA, Europe, Asia, Africa and Latin America, respectively. The other potential modifying factors, including sample resource and adjustment for HPV, AFI or reproductive factors, had no significant influence on the estimates.

No obvious publication bias was found by the analysis using funnel plot, Egger's test (P value for bias=0.319), and Begg's test ( $\mathrm{P}$ value for bias $=0.785)$. The sensitivity analysis revealed that the overall ORs remained significant and ranged from $1.68(95 \% \mathrm{CI} 1.42-91.4)$ to $1.82(95 \% \mathrm{CI}$ $1.50-2.15$ ) by removing one study at a time from the main analysis. The summery OR altered slightly after excluding the study with high risk individuals (Rostad, Schei and Da, 2003; Kanjanavirojkul et al., 2006) (OR=1.76, 95\%CI 1.48-2.04). In the studies that had adjusted for HPV infection, omission one study at a time did not alter the summary risk estimate.

We observed a linear relationship between the number of sexual partners and ICC ( $\mathrm{P}$ for non-linearity $<0.001$; Figure 5A). As showed at Figure 5A, the degree of increase appeared to be more obvious when there were more than 4-5 sexual partners. Non-linearity relation was found in the studies that did not adjust for HPV infection (P for non- 
linearity $<0.001$; Figure 5B), and the trend was similar with the overall result. However, when we explored the relation in the studies that had controlled for HPV infection, we observed a linear relation ( $\mathrm{P}$ for linearity $<0.001$; Figure $5 \mathrm{C}$ ) between the number of sexual partners and ICC. The risk of ICC increased as the number of sexual partners increased.

\section{Discussion}

From the result of this meta-analysis, we observed a significant increased risk of cervical diseases in individuals with multiple sexual partners compared to individuals with few partners, both in non-malignant cervical disease and in ICC. The association remained exist even after controlling for the status of HPV infection, which is a major cause of cervical cancer. Notably, the frequency-risk relationship analysis showed that the trend of increased risk varied after controlling for the status of HPV infection. However, the risk for either non-malignant cervical disease or ICC increased as the number of sexual partners increased.

In the non-malignant cervical disease, we found the risk estimate for case-control studies was higher than that of the cohort studies. Selection and recall bias may be partly responsible for this difference. In addition, the association between cervical cancer and the number of sexual partners varied after adjustment for HPV infection, indicating that HPV infection was a confounding factor. However, their risk estimates were both statistically significant. Concerning the frequency-risk relation, it was worth noting that the trend of risk changed after controlling for HPV infection, implying that the effect of HPV infection has to be considered.

In the frequency-risk relation of the number of sexual partners and ICC, the estimates of the studies unadjusted for HPV infection were very similar to the results of previous collaborative reanalysis (2009), which had conditioned on age, study or study centre and AFI. Interestingly, we found that the association between the number of sexual partners and ICC was differed by geographical region. Previous reanalysis (2009) did not analyze whether there was heterogeneity existed in different regions. However, the pooled analysis of IARC (Vaccarella et al., 2006) had reported that the risk of HPV infection for multiple sexual partners in Spain was higher, which also implied that there might be a regional difference in the risk of HPV infection. This finding was very similar to our result of regional difference. Considering that the association between HPV infection and cervical cancer was very strong, we assumed that the regional difference of ICC might mainly be caused by the risk for HPV infection for multiple partners. For this reason, we can not ignore the influence of HPV infection. Previous reanalysis (2009) reported that the estimate was lower in hospital-based case-control studies than that in population-based casecontrol studies, and they considered that this heterogeneity was attributable to the higher number of sexual partners reported by women in population-based case-control studies than that in hospital-based studies. However, we found that this difference was not statistically significant by using meta-regression in our study. And we observed that most of the population-based case-control studies were performed in Europe and USA, while most of the hospital-based case-control studies were performed in other regions. Thus, the higher number of sexual partners reported in Europe and USA might also explain some of the regional difference of ICC.

There are several potential biological mechanisms that can explain the observed results. Firstly, the pronounced risk of cervical cancer associated with the number of sexual partners is generally ascribed to the increase risk of HPV infection (Kjaer et al., 1996b). In this present meta-analysis, we found that the association remained significant even after controlled for HPV infection. It means that there might be other unknown factors that contributed to the association between the number of sexual partners and cervical cancer. Some studies indicated that males play a critical role in the risk of developing cervical cancer in women (Buckleyet al., 1981; Zunzunegui, et al., 1986). Nevertheless, the male factor is most likely linked to the risk of HPV infection (Yoo et al., 1997). On the other hand, other studies suggested that the number of sexual partners could be an independent factor of cervical cancer (Parazzini et al., 1988). There were also studies suggested that other sexually transmitted viruses, such as herpes simplex virus 2 (HSV-2) (Sitas et al., 2000) and human immunodeficiency virus (HIV) might increase the risk of cervical cancer (Castellsague et al., 2006). Previous studies had confirmed the positive relationship between the number of sexual partners and the infection of these viruses (Malamba et al., 1994; Vaccarella et al., 2006). It is unclear whether the association is due to an independent risk or whether it is indirectly mediated by other common risk factors, such as HIV infection and HSV-2 infection. We were unable to perform analyses stratified the adjustments for HIV or HSV-2 infection because there was no eligible study adjusted for them. Some studies indicated that AFI and reproductive factors could be confounding factors $(2006 ; 2009)$. In our metaanalysis, we did not observe significant difference between them. We found few studies focused on the age at first pregnancy and the number of pregnancies simultaneously, especially in those with non-malignant cervical disease. Therefore it is challenging to accurately evaluate the cancer risk associated with the number of sexual partners.

There are several potential limitations in this metaanalysis. First, the acceptability and reliability of questions on sexual behaviors have always been of great concern (BOYD and DOLL, 1964). Women usually tend to conceal the number of their sexual partners (Schroder, Carey and Vanable, 2003), particularly in conservative and traditional regions. From the result of non-malignant cervical disease, we did not observe an obvious regional disparity. Therefore it is difficult to interpret the question of reliability. On the other hand, it was hard to explain why there was no regional disparity in non-malignant cervical disease. Some studies reported that the association of ICC and HPV infection was much stronger than the relation of CIN and HPV infection (Kim et al., 2012). We considered that ICC might be more likely to be affected by HPV. Similarly, the trend of frequency-risk relation between ICC and the number of sexual partners 
had become more obvious after controlling for HPV infection. Secondly, the data we obtained did not allow us to explore the relationship in different stages of ICC. Neither had it allowed us to investigate whether there is a difference between squamous cell carcinoma and adenocarcinoma. In the previous reanalysis (2009), no evidence was found to prove that they were different. Additionally, the sensitivity might not be uniform in different HPV testing method. We found that most studies used PCR to detect HPV-DNA, which is the most sensitive method (Herrington, 1999). No obvious heterogeneity was found in the studies that were adjusted for HPV infection. Finally, we did not include the studies restricted to HPVpositive women as previously reanalysis did. This might possibly affect the result of analysis. However, restriction to HPV-positive women in the study might affect the comparability between case and control because the HPV infection in control may be a recently acquired transient infection, while the infection in case could be a long-term infection. In addition, restriction to HPV-positive women will not reflect the whole population. For this reason, our study with no restriction to HPV-positive or HIV-positive women is more appropriate.

In conclusion, based on the results of this metaanalysis, we suggested that the number of sexual partners was associated with the development of cervical cancer. The association remained significant after controlling for HPV infection. It indicated that women with multiple sexual partners are the high risk population of cervical cancer even the HPV vaccines are implemented in a wide population. Further study is needed to collect and evaluate more detailed information on HIV, HSV-2 infection and other reproductive factors before concluding that the number of sexual partners is an independent risk factor for cervical cancer.

\section{Acknowledgements}

This work was supported by fund from the Guangdong key laboratory of molecular epidemiology (no. 2010 A060801015) and the International cooperation project (no. 2011 J5200017). The funders had no role in study design, data collection and analysis, and interpretation of the data.

\section{References}

(2006). Cervical carcinoma and reproductive factors: collaborative reanalysis of individual data on 16,563 women with cervical carcinoma and 33,542 women without cervical carcinoma from 25 epidemiological studies. INT J CANCER, 119, $1108-24$.

(2009). Cervical carcinoma and sexual behavior: collaborative reanalysis of individual data on 15,461 women with cervical carcinoma and 29,164 women without cervical carcinoma from 21 epidemiological studies. Cancer Epidemiol Biomarkers Prev, 18, 1060-9.

Begg CB, Mazumdar M (1994). Operating characteristics of a rank correlation test for publication bias. BIOMETRICS, 50, 1088-101.

Bosch FX, Munoz N, de Sanjose S, et al (1992). Risk factors for cervical cancer in Colombia and Spain. INT J CANCER,
52, 750-8.

Boyd JT, Doll R (1964). A study of the aetiology of carcinoma of the cervix uteri. Br J Cancer, 13, 419-34.

Brisson J, Morin C, Fortier M, et al (1994). Risk factors for cervical intraepithelial neoplasia: differences between lowand high-grade lesions. AM J EPIDEMIOL, 140, 700-10.

Brisson J, Roy M, Fortier M, et al (1988). Condyloma and intraepithelial neoplasia of the uterine cervix: a case-control study. AM J EPIDEMIOL, 128, 337-42.

Buckley JD, Harris RW, Doll R, et al (1981). Case-control study of the husbands of women with dysplasia or carcinoma of the cervix uteri. LANCET, 2, 1010-5.

Castellsague X, Diaz M, de Sanjose S, et al (2006). Worldwide human papillomavirus etiology of cervical adenocarcinoma and its cofactors: implications for screening and prevention. J Natl Cancer Inst, 98, 303-15.

Coker AL, Rosenberg AJ, McCann MF, et al (1992). Active and passive cigarette smoke exposure and cervical intraepithelial neoplasia. Cancer Epidemiol Biomarkers Prev, 1, 349-56.

Egger M, Davey SG, Schneider M, et al (1997). Bias in metaanalysis detected by a simple, graphical test. $B M J, \mathbf{3 1 5}$, 629-34.

Hernandez-Hernandez DM, Ornelas-Bernal L, Guido-Jimenez M, et al (2003). Association between high-risk human papillomavirus DNA load and precursor lesions of cervical cancer in Mexican women. GYNECOL ONCOL, 90, 310-7.

Herrero R, Brinton LA, Reeves WC, et al (1990). Sexual behavior, venereal diseases, hygiene practices, and invasive cervical cancer in a high-risk population. CANCER-AM CANCER SOC, 65, 380-6.

Herrington CS (1999). Do HPV-negative cervical carcinomas exist?--revisited. J PATHOL, 189, 1-3.

Higgins JP, Thompson SG (2002). Quantifying heterogeneity in a meta-analysis. STAT MED, 21, 1539-58.

Higgins JP, Thompson SG, Deeks JJ, et al (2003). Measuring inconsistency in meta-analyses. BMJ, 327, 557-60.

Kanjanavirojkul N, Pairojkul C, Yuenyao P, et al (2006). Risk factors and histological outcome of abnormal cervix with human papilloma infection in northeastern Thai-women. Asian Pac J Cancer Prev, 7, 567-70.

Kim J, Kim BK, Lee CH, et al (2012). Human papillomavirus genotypes and cofactors causing cervical intraepithelial neoplasia and cervical cancer in Korean women. INT $J$ GYNECOL CANCER, 22, 1570-6.

Kjaer SK (1998). Risk factors for cervical neoplasia in Denmark. APMIS Suppl, 80, 1-41.

Kjaer SK, van den Brule AJ, Bock JE, et al (1996). Human papillomavirus--the most significant risk determinant of cervical intraepithelial neoplasia. INT J CANCER, 65, 601-6.

Kjellberg L, Hallmans G, Ahren AM, et al (2000). Smoking, diet, pregnancy and oral contraceptive use as risk factors for cervical intra-epithelial neoplasia in relation to human papillomavirus infection. Br J Cancer, 82, 1332-8.

Malamba SS, Wagner HU, Maude G, et al (1994). Risk factors for HIV-1 infection in adults in a rural Ugandan community: a case-control study. AIDS, 8, 253-7.

McDougall JA, Madeleine MM, Daling JR, et al (2007). Racial and ethnic disparities in cervical cancer incidence rates in the United States, 1992-2003. Cancer Causes Control, 18, 1175-86.

Millikan RC (1994). Epidemiologic evidence showing that human papillomavirus infection causes most cervical intraepithelial neoplasia. J Natl Cancer Inst, 86, 392-3.

Moher D, Liberati A, Tetzlaff J, et al (2010). Preferred reporting items for systematic reviews and meta-analyses: the PRISMA statement. INT J SURG, 8, 336-41.

Olsen AO, Dillner J, Gjoen K, et al (1996). A population- 


\section{Zhi-Chang Liu}

based case-control study of human papillomavirus-type-16 seropositivity and incident high-grade dysplasia of the uterine cervix. INT J CANCER, 68, 415-9.

Orsini N, Li R, Wolk A, et al (2012). Meta-analysis for linear and nonlinear dose-response relations: examples, an evaluation of approximations, and software. AM J EPIDEMIOL, 175, 66-73.

Parazzini F, La Vecchia C, Negri E, et al (1988). Risk factors for adenocarcinoma of the cervix: a case-control study. $\mathrm{Br}$ J Cancer, 57, 201-4.

Parazzini F, La Vecchia C, Negri E, et al (1992). Risk factors for cervical intraepithelial neoplasia. CANCER-AM CANCER SOC, 69, 2276-82.

Rostad B, Schei B, Da CF (2003). Risk factors for cervical cancer in Mozambican women. Int J Gynaecol Obstet, 80, 63-5.

Schroder KE, Carey MP, Vanable PA (2003). Methodological challenges in research on sexual risk behavior: II. Accuracy of self-reports. Ann Behav Med, 26, 104-23.

Sitas F, Pacella-Norman R, Carrara H, et al (2000). The spectrum of HIV-1 related cancers in South Africa. INT J CANCER, 88, 489-92.

Thomas DB, Qin Q, Kuypers J, et al (2001). Human papillomaviruses and cervical cancer in Bangkok. II. Risk factors for in situ and invasive squamous cell cervical carcinomas. AM J EPIDEMIOL, 153, 732-9.

Vaccarella S, Franceschi S, Herrero R, et al (2006). Sexual behavior, condom use, and human papillomavirus: pooled analysis of the IARC human papillomavirus prevalence surveys. Cancer Epidemiol Biomarkers Prev, 15, 326-33.

Velema JP, Ferrera A, Figueroa M, et al (2002). Burning wood in the kitchen increases the risk of cervical neoplasia in HPVinfected women in Honduras. INT J CANCER, 97, 536-41.

Walboomers JM, Jacobs MV, Manos MM, et al (1999). Human papillomavirus is a necessary cause of invasive cervical cancer worldwide. J PATHOL, 189, 12-9.

Wang PD, Lin RS (1996). Risk factors for cervical intraepithelial neoplasia in Taiwan. GYNECOL ONCOL, 62, 10-8.

Ye X, Fu J, Yang Y, et al (2013). Dose-risk and duration-risk relationships between aspirin and colorectal cancer: a metaanalysis of published cohort studies. PLOS ONE, 8, e57578.

Yoo KY, Kang D, Koo HW, et al (1997). Risk factors associated with uterine cervical cancer in Korea: a case-control study with special reference to sexual behavior. J EPIDEMIOL, 7, 117-23.

Zunzunegui MV, King MC, Coria CF, et al (1986). Male influences on cervical cancer risk. AM J EPIDEMIOL, $123,302-7$. 